RESEARCH NOTE

\section{Influence of the Route of Administration of Pig-serum in the Induction of Hepatic Septal Fibrosis in Rats}

\section{Zilton A Andrade $/^{+}$, Adriana Godoy*}

Laboratório de Patologia Experimental, Centro de Pesquisas Gonçalo Moniz-FIOCRUZ, Rua Valdemar Falcão 121, 40945-001 Salvador, BA, Brasil

Key words: liver - septal fibrosis - fibrogenesis pig-serum model

Repeated intraperitoneal injections of pig-serum into rats result in the production of hepatic septal fibrosis (F Paronetto \& H Popper 1966 Am $J$ Pathol 40: 1087-1101). This is an interesting model of liver fibrosis that occurs without previous liver-cell necrosis or inflammation (E Rubin et al. 1968 Am J Pathol 52: 111-119). Its pathogenesis primarily involves the sinusoidal Kupffer cell/perisinusoidal Ito-cell axis, which is activated by so-called fibrogenic cytokines (AM Gressner \& MG Bachem 1990 Sem Liver Dis 10: 30-46, EJ Kovacs 1991 Immunol Today 12: 17-23). Recently septal fibrosis of the liver was detected as an important manifestation of Capillaria hepatica infection in rats (LA Frerreira \& ZA Andrade $1993 \mathrm{Mem}$ Inst Oswaldo Cruz 88: 441-447). This finding, as well as similar ones seen in schistosomiasis (AW Cheever \& Andrade ZA 1970 Gaz Med Bahia 70: 67-74) and fascioliasis (JD Dargie et al. 1974 Parasitic Zoonoses, Clinical and Experimental Studies, Academic Press, New York) are suggestive that antigens being slowly liberated within the liver itself would be more readily available to the hepatic intra and peri-sinusoidal fibrogenic system. Thus, the closer the appropriate antigen releasing is to the liver, the more effective would be its ability to induce septal fibrosis. Actually little is known about the influence of the route of antigen administration for the experimental generation of septal fibrosis of the liver. To investigate this subject two different routes of antigen administration

${ }^{+}$Corresponding author. Fax: +55-71-359.4292

*Fellow, Brazilian National Research Council (CNPq) Received 10 April 1996

Accepted 25 June 1996 were investigated during the production of pig-serum induced septal fibrosis of the liver in rats. Twenty Wistar rats were used. They were of both sexes, weighing 170/300g, maintained in separate cages with a commercial balanced diet and water ad libitum. Pig blood was collected from several animals in a slaughter house. After coagulation it was immediately centrifuged for the collection of serum, which was stored in small vials at $4^{\circ} \mathrm{C}$. Electrophoresis of pig-serum revealed: albumin: $48.7 \%$, $\alpha 1: 0.5 \%, \alpha 2: 20.0 \%, \beta 1: 3.0 \%, \beta 2: 4.5 \%$ and $\gamma$ : $23.3 \%$. It has been determined that the albumin fraction is the one mainly related to the fibrogenic activity of the pig-serum in rats (Paronetto \& Popper loc. cit.). Serum injections were made twice a week for a total of 26 injections. Half of the animals was injected intraperitoneally and the other half subcutaneously in the dorsal region. All animals completed the entire treatment period without losing weight or showing any external sign of disease. Observation of fibrosis was performed in sections from the liver taken after autopsy of the animals. Fragments of the liver were fixed in $\mathrm{pH}$ 7.4 buffered $10 \%$ formalin, embedded in paraffin and the sections stained with hematoxylin and eosin, the picro-sirius-red method for collagen and the Gomori silver impregnation method for reticulum. Grossly there was only a mild thickening of the external capsule and a slight increase in consistency in a few of the livers taken from the intraperitoneally-injected animals. Microscopically, septal fibrosis, represented by fine, long and straight lines of fibrous tissue located along the zone III of the hepatic acinus was detected in $42.8 \%$ of the animals intraperitoneally injected with pigserum. In the other animals of this same group variable degrees of perisinusoidal thickening and of hyperplasia of reticulin fibrils were detected in all but two of them.

The livers of the animals injected into the subcutaneous dorsal region with pig-serum were essentially normal and not even perisinusoidal reticulin increase was detected in them. Thus, clearcut results pointed to the influence of the route of administration of pig-serum in the experimental production of septal liver fibrosis in rats. Reasons for this have not been determined. Of course it is not merely a question of a distance to be traveled by the factor(s) in the pig-serum, but may involve a host of complex influences, one of them being differences in the kind of local antigen-presenting cells and the way local tissues process foreign material. The presence of septal fibrosis in $40 \%$ of the animals injected by the intraperitoneal route represents the usual percentage obtained by others with the rat pig-serum model (K Fujiwara et al. 1988 Hepatology 8: 804-807, ZA Andrade 1991 Int J Exp Path 72: 551-562). 
